Check for updates

Cite this: Chem. Sci., 2019, 10, 5678

๑ All publication charges for this article have been paid for by the Royal Society of Chemistry

\title{
On the encapsulation and assembly of anticancer drugs in a cooperative fashion $\uparrow$
}

\author{
Weikun Wang, ${ }^{a}$ Han Wang, ${ }^{b}$ Lei Zhiquan, ${ }^{a}$ Han Xie, ${ }^{a}$ Honggang Cui ${ }^{b}$ \\ and Jovica D. Badjić (D) *a
}

In this study, we report the remarkable recognition and assembly characteristics of $D_{3 h}$ symmetric basket $1^{6-}$ containing two adjoining and nonpolar cavities with six biocompatible GABA residues at their northern and southern termini. From the results of experimental $\left({ }^{1} \mathrm{H} N M R\right.$, fluorescence and UV-Vis spectroscopies) and computational (MM-MC/OPLS3e) investigations, we deduced that hexaanionic $1^{6-}$ captured two molecules of anticancer drug doxorubicin $2^{+}$in water and accommodated them in its two deep cavities. The formation of stable $1^{6-} \subset 2_{2}{ }^{2+}\left(K_{a}=3 \times 10^{12} \mathrm{M}^{-2}\right)$ was accompanied by the exceptional homotopic cooperativity $\left(\alpha=4 K_{2} / K_{1}=112\right)$ in which $K_{1}=3.2 \pm 0.8 \times 10^{5} \mathrm{M}^{-1}$ and $K_{2}=9 \pm$ $1 \times 10^{6} \mathrm{M}^{-1}$. Furthermore, bolaamphiphilic $1^{6-} \subset 2_{2}{ }^{2+}$ assembled into spherical nanoparticles (DLS, cryoTEM and TEM) possessing $41 \%$ drug loading. The preorganization of abiotic receptor $1^{6-}$ and its complementarity to $2^{+}$have been proposed to play a part in the positive cooperativity in which ten favorable noncovalent contacts (i.e. hydrogen bonds, salt bridges, $\mathrm{C}-\mathrm{H} \cdots \pi$ and $\pi-\pi$ contacts) are formed between doxorubicin and the dual-cavity host. In the case of topotecan $3^{+}$, however, the absence of multiple and favorable basket $\subset$ drug interactions resulted in the predominant formation of a binary $1^{6-} \subset 3^{+}$complex $\left(K_{1}=2.12 \pm 0.01 \times 10^{4} \mathrm{M}^{-1}\right)$ and the negative homotopic allostery $(\alpha \ll 1)$. To summarize, our study lays out a roadmap for creating a family of novel, accessible and multivalent hosts capable of complexing anticancer agents in a cooperative manner. As basket $\subset$ drug complexes organize into highly loaded nanoparticles, the reported soft material is amenable to the bottom-up construction of stimuli-responsive nanomedicine capable of effective scavenging and/or delivery of drugs.

Received 20th March 2019

Accepted 1st May 2019

DOI: $10.1039 /$ c9sc01380f

rsc.li/chemical-science

\section{Introduction}

Nanosystems carrying anticancer drugs, i.e. nanomedicine, ${ }^{\mathbf{1}}$ hold great promise in the area of targeted chemotherapy for selective detection and extermination of cancer cells. ${ }^{\mathbf{a}, \mathbf{2}}$ In particular, drug-delivery nanoparticles ${ }^{3}$ extravasate from abnormal blood vessels into the tumor microenvironment (EPR effect), ${ }^{4}$ which with their surface modification could improve the therapy. ${ }^{3,5,6}$ Besides, stimuli-responsive and biocompatible ${ }^{7}$ cyclodextrins, ${ }^{8}$ calixarenes ${ }^{9}$ and cucurbiturils ${ }^{10}$ are also capable of transporting/releasing anticancer drugs. ${ }^{11}$ As a result of the inclusion complexation, ${ }^{12}$ abiotic hosts are expected to improve the therapy by allowing spatiotemporal control ${ }^{13}$ of drug release and also assisting with (a) loading capacity, ${ }^{14}$ (b) solubility, ${ }^{15}$ (c) bioavailability ${ }^{16}$ and (d) stability of drugs. ${ }^{17}$ In addition to such virtues, allosteric and chelate cooperativity ${ }^{\mathbf{1 8}}$ operating in the

\footnotetext{
${ }^{a}$ Department of Chemistry \& Biochemistry, The Ohio State University, 100 West 18th Avenue, Columbus, $\mathrm{OH} 43210$, USA

${ }^{b}$ Department of Chemical and Biomolecular Engineering, The Johns Hopkins University, Maryland Hall 221, 3400 North Charles Street, Baltimore, MD 21218, USA $\dagger$ Electronic supplementary information (ESI) available: The preparation and characterization of $\mathbf{1}$ with additional experimental and computational details. See DOI: $10.1039 / \mathrm{c} 9 \mathrm{sc} 01380 \mathrm{f}$
}

host-guest complexation of pharmaceuticals is expected to give rise to an amplified response so that small changes in the external signal (i.e. stimulus) trigger a large non-linear outcome. ${ }^{19}$ The on/off mode ${ }^{20}$ of delivery is, however, challenging to attain ${ }^{21}$ yet a sought-after strategy to achieve minimal release of the payload before reaching its targeted site followed by a burst in drug release. ${ }^{22}$

Recently, we reported about dual-cavity baskets comprising $\operatorname{six}^{23}(S)$ alanine residues at their periphery (Fig. 1) and acting in an allosteric manner ${ }^{24}$ by trapping small molecules akin to nerve agents. ${ }^{25}$ As these complexes assembled into vesicles, ${ }^{25 b}$ we became increasingly curious about examining the capacity of deeper dual-cavity $\mathbf{1}^{6-}$ (Fig. 1A) for including anticancer drugs doxorubicin $2^{+}$(DOX) and topotecan $3^{+}$in its two adjoining cavities. At first, the inner space of basket $\mathbf{1}^{6-}$ seemed complementary ${ }^{26}$ to $2^{+}$and $3^{+}$in size, shape and polarity (Fig. 1A). Moreover, structurally unique $\mathbf{1}^{6-}$ possesses six biocompatible GABA ( $\gamma$-aminobutyric acid) residues at its southern and northern termini for acting as "sticky" carboxylate fingers capable of grabbing functional drug molecules and holding them in two aromatic pockets. ${ }^{27}$ The results of our experimental and computational studies have revealed a fascinating way by which dual-cavity $\mathbf{1}^{6-}$ encapsulates two molecules 
(A)

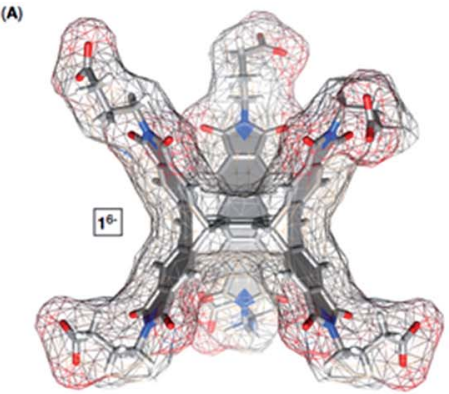

(B)

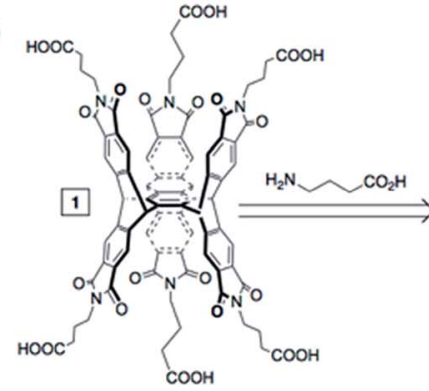

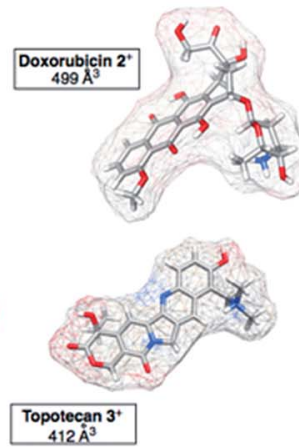

$412 A^{3}$ (c)

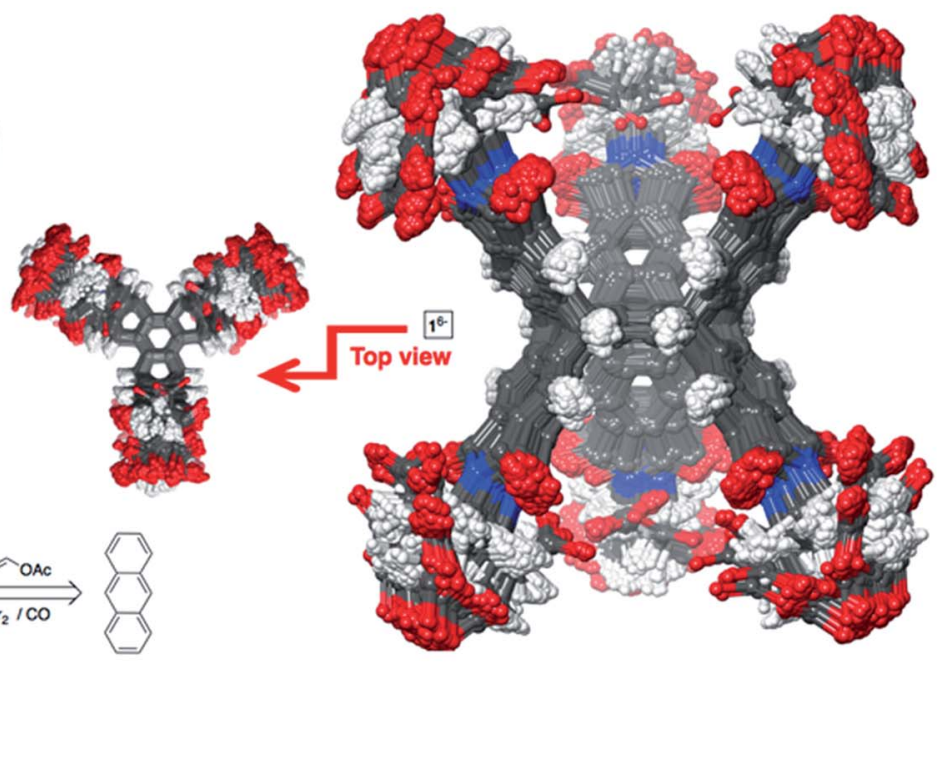

Fig. 1 (A) van der Waals surfaces of energy-minimized basket $1^{6-}$, doxorubicin $2^{+}$and topotecan $3^{+}$(OPLS3e, Maestro). (B) Basket $1^{6-}$ was prepared from anthracene, vinyl acetate and GABA (Scheme S1 $\dagger$ ). (C) Top (left) and side (right) views of the aligned conformers of $1^{6-}(<1.5 \mathrm{kcal}$ $\mathrm{mol}^{-1}$ ) obtained from the Monte-Carlo conformational search (OPLS3e, Maestro) in implicit water.

of doxorubicin $2^{+}$with exceptional homotopic cooperativity of $\alpha=4 K_{2} / K_{1}=112 !^{18,28}$ Moreover, stable ternary $\mathbf{1}^{6-} \subset 2_{2}{ }^{2+}$ complexes $\left(K_{\mathrm{a}}=10^{12} \mathrm{M}^{-2}\right)$ assembled into spherical nanoparticles possessing a high (41\%) drug loading. ${ }^{29}$

\section{Results and discussion}

Dual-cavity 1 (Fig. 1B) was prepared from inexpensive anthracene, vinyl acetate and GABA by following a recently developed procedure (Scheme S1 $\dagger$ ). ${ }^{30}$ After basket 1 was dissolved in $10 \mathrm{mM}$ phosphate buffer (PBS) at $\mathrm{pH}=7.0$, its ${ }^{1} \mathrm{H}$ NMR spectrum showed a set of five, somewhat broad, resonances corresponding to, on average, $D_{3 \mathrm{~h}}$ symmetric $\mathbf{1}^{6-}$ (Fig. 2A); note that for six remote carboxylates, we assumed $\mathrm{p} K_{\mathrm{a}}<5 .^{31}$ In line with ${ }^{1} \mathrm{H}$ NMR results, the Monte-Carlo conformational sampling of $\mathbf{1}^{6-}$, in implicit water solvent (OPLS3e, Maestro), revealed numerous conformers (Fig. 1C) populating the equilibrium $\left(<1.5 \mathrm{kcal} \mathrm{mol}^{-1}\right)$. In solution, these conformers were likely to exchange at a high rate to give the observed ${ }^{1} \mathrm{H}$ NMR spectrum. Furthermore, six carboxylates cluster at the outer side of $\mathbf{1}^{6-}$ to make the binding pockets deep, nonpolar and suitable for accommodating nonpolar segments of doxorubicin $2^{+}$and topotecan $3^{+}$(Fig. 1A). The phthalimide side arms are "bouncing" back and forth to alter the cavity size, ${ }^{32}$ which is central to the induced-fit mode of complexation of the first molecule of drug, as discussed in the text below. ${ }^{33}$

Variously concentrated solutions of $\mathbf{1}^{6-}$ exhibited a sharp change in the UV-Vis extinction coefficient $\left(\varepsilon_{300}\right.$, i.e. slopes in Fig. $2 \mathrm{~B}$ ) of this chromophore at $\sim 0.12 \mathrm{mM}^{21}$ The "segmental adherence to the Lambert-Beer law suggested that the basket transitioned from the monomeric to the aggregated state at 0.12
$\mathrm{mM}$ thereby depicting its critical aggregation concentration (CAC) ${ }^{25 b, 34}$ Indeed, the results of dynamic light scattering measurements of $0.76 \mathrm{mM} \mathrm{1}^{6-}$ (DLS, Fig. 2C) were in line with the formation of nanosized particles, while the Tyndall effect was apparent for $0.4 \mathrm{mM}$ but absent for the $0.05 \mathrm{mM}$ solution of $\mathbf{1}^{6-}$ (Fig. 2C). At last, cryo-TEM and TEM imaging (Fig. 2D) revealed the formation of circa $40-50 \mathrm{~nm}$ spherical objects possessing uniform coloration with no distinguishable features (i.e. double layer). Accordingly, bolaamphiphilic $\mathbf{1}^{6-}$ is likely populating the interior of the spherical aggregates with a packing mode that still needs to be elucidated. ${ }^{34 a}$

After an incremental addition of doxorubicin $2^{+}$to basket $\mathbf{1}^{6-}$ (Fig. 2A), there followed a broadening of ${ }^{1} \mathrm{H}$ NMR resonances from the host while signals corresponding to the drug's nuclei became ill defined. In particular, $\mathbf{H}_{\mathrm{A}}-\mathbf{H}_{\mathrm{D}}$ signals from the doxorubicin's methoxybenzene (shown in red, Fig. 2A) were difficult to resolve, as they were likely becoming magnetically shielded and broadening into the baseline. Alternatively, $\mathbf{H}_{\mathrm{E}^{-}}$ $\mathbf{H}_{\mathrm{N}}$ signals from the doxorubicin's non-aromatic side (shown in blue, Fig. 2A) were easier to identify by comparing the spectra. From the NMR results, we hypothesized that doxorubicin $2^{+}$ formed a stable host-guest complex with $\mathbf{1}^{6-}$ to result in the complexation dynamics occurring at an intermediate rate and/ or rapid $T_{2}$ relaxation of $\mathbf{H}_{\mathrm{A}}-\mathbf{H}_{\mathrm{D}}$ protons $\mathbf{2}^{+}$inside the cavity(ies) of $\mathbf{1}^{6-} \cdot{ }^{35}$ As the magnetic environment and dynamics of $\mathbf{H}_{\mathrm{E}}-\mathbf{H}_{\mathrm{N}}$ were perturbed to a lesser degree than $\mathbf{H}_{\mathrm{A}}-\mathbf{H}_{\mathrm{D}}$ protons, however, these nuclei stayed away from the concave aromatic pockets of $\mathbf{1}^{6-}$. To further quantify the inclusion complexation, we noted that incremental addition of basket $1^{6-}$ to $2^{+}$(Fig. 3A) quenched the emission from doxorubicin; ${ }^{36}$ the quenching was static, ${ }^{37}$ since in the absence of the complex formation the emission from the drug remained steady (Fig. S7†). The binding 
(A)

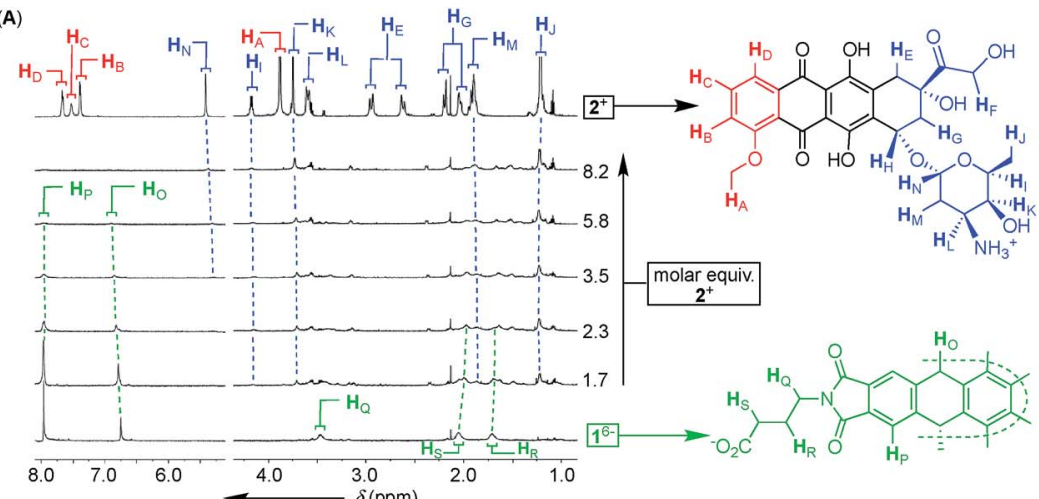

(C)

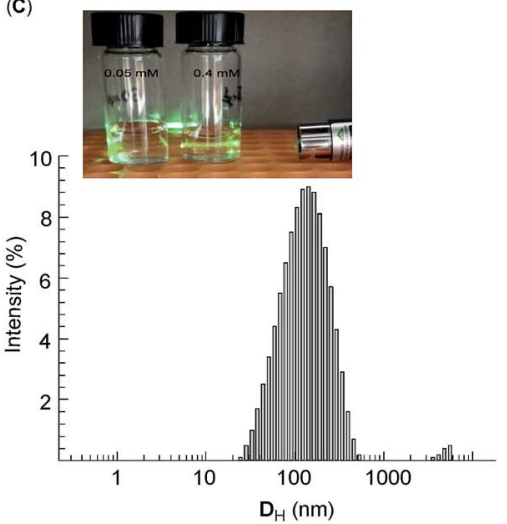

(B)

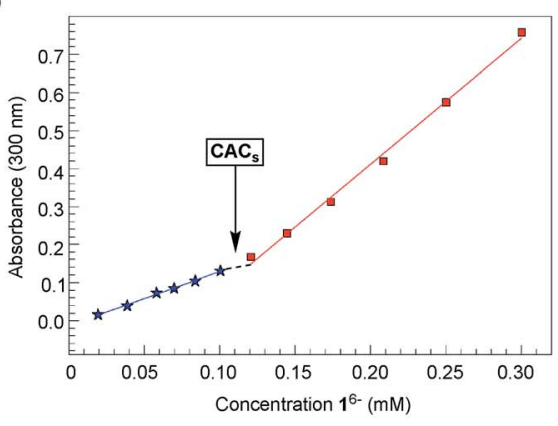

(D)
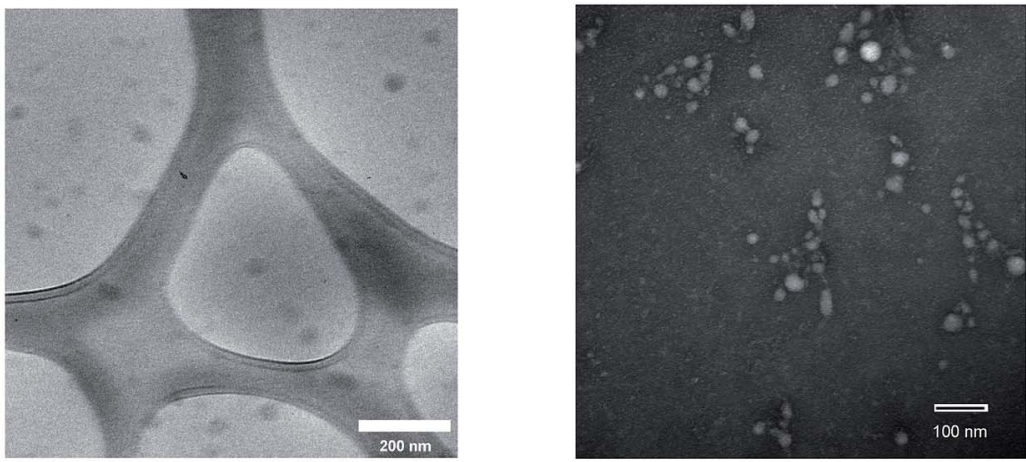

Fig. 2 (A) ${ }^{1} \mathrm{H}$ NMR spectra $\left(600 \mathrm{MHz}, 298 \mathrm{~K}\right.$ ) of $0.04 \mathrm{mM}$ basket $1^{6-}$ in $10 \mathrm{mM}$ PBS at $\mathrm{pH}=7.0$ (bottom, green) before and after incremental addition of doxorubicin $2^{+}$(top, blue-red). (B) UV-Vis absorbance $\left(300 \mathrm{~nm}\right.$ ) of differently concentrated solutions of $1^{6-}$ in $10 \mathrm{mM} \mathrm{PBS}$ at pH $=7.0$ as a function of its concentration. Each set of data (blue and red) was fit to a linear equation $\left(R^{2}>0.99\right.$, SigmaPlot) using the least-square regression analysis. (C) The Tyndall effect can be seen for $0.4 \mathrm{mM}$ (right) but not for a $0.05 \mathrm{mM}$ (left) solution of $1^{6-}$. The size distribution of particles in a $0.76 \mathrm{mM}$ solution of $1^{6-}(10 \mathrm{mM}$ PBS at $\mathrm{pH}=7.0)$ obtained from DLS measurement at 298.0 K. (D) Cryo-TEM image (left) of $0.6 \mathrm{mM}$ solution of basket $1^{6-}$ in $10 \mathrm{mM} \mathrm{PBS}$ at $\mathrm{pH}=7.0$ and TEM image (right) of $0.2 \mathrm{mM} 1^{6-}$ in $10 \mathrm{mM} \mathrm{PBS}$ at pH $=7.0$ deposited on a copper grid and stained with uranyl acetate.

isotherm (Fig. 3B; see also Fig. S8 $\dagger$ and www.supramolecular.org) fits well to the $1: 2$ complexation mode ${ }^{28}$ with a random distribution of residuals ${ }^{38}$ and $K_{1}=3.2 \pm$ $0.8 \times 10^{5} \mathrm{M}^{-1} / K_{2}=9 \pm 1 \times 10^{6} \mathrm{M}^{-1}$. The method of continuous variation (UV-Vis, Fig. 3C) was also in line with the formation of ternary $1^{6-} \subset 2_{2}{ }^{2+}$ with the parabolic function peaking at $\left[2^{+}\right] /$ $\left(\left[1^{6-}\right]+\left[2^{+}\right]\right) \sim 0.67 .^{38}$ The positive cooperativity of $\alpha=4 K_{2} / K_{1}=$ 112 is large and quite unusual for an artificial host. ${ }^{21,39} \mathrm{We}$ therefore suspected that the formation of ternary $\mathbf{1}^{6-} \subset \mathbf{2}_{2}{ }^{2+}$ could be accompanied by an aggregation of these bolaamphiphilic complexes so that the observed cooperativity is in part arising from the chelate (multivalent) cooperativity. ${ }^{18}$ In support of such logic, the observed broadening of ${ }^{1} \mathrm{H}$ NMR signals of both $1^{6-}$ and $2^{+}$in Fig. 2A could have, in part, resulted from their aggregation. First, DLS measurements of $1^{6-} \subset 2_{2}{ }^{2+}$ showed the presence of circa $60-240 \mathrm{~nm}$ particles (Fig. 3D) having electrokinetic (zeta) potential of $\zeta=-33.6$ (Fig. S9†) and therefore moderate stability. ${ }^{25 b}$ Likewise, TEM (Fig. 3E) and cryo-TEM imaging (Fig. 3F) revealed the formation of nanoparticles whose size (circa $50 \mathrm{~nm}$ ), shape and, perhaps, assembly mode were akin to those forming from $\mathbf{1}^{6-}$. As nanoparticles are composed of dual-cavity hosts $\left(M_{\mathrm{w}}=1536\right)$ with each holding two molecules of doxorubicin $\left(M_{\mathrm{w}}=2 \times 544=1088\right)$, it follows that the material's loading is circa $41 \%$, which is highly desirable ${ }^{13}$ for nanomedicine. ${ }^{1 a}$

Encouraged by the results with DOX $2^{+}$, we turned to examine the entrapment of another therapeutic anticancer drug, topotecan $3^{+}$(Fig. 4). This flat molecule is in size and shape similar to doxorubicin (Fig. 1A) therefore making a good candidate for occupying the binding pockets of $\mathbf{1}^{6-}$. Upon an incremental addition of a standard solution of $3^{+}$to $1^{6-}$, there were small but steady magnetic perturbations of ${ }^{1} \mathrm{H}$ NMR resonances from the basket (green in Fig. 4A). In particular, a greater proportion of $3^{+}$resulted in the $\mathbf{H}_{\mathrm{L}}$ singlet from $D_{3 \mathrm{~h}}$ symmetric $\mathbf{1}^{6-}$ turning into a doublet. The observation was taken as a sign depicting the predominant formation of pseudo $C_{3 \mathrm{v}}$ symmetric $1^{6-} \subset 3^{+}$in which a single cavity of the host is holding the drug. Furthermore, $\mathbf{H}_{\mathrm{A} / \mathrm{B} / \mathrm{C}}$ and $\mathbf{H}_{\mathrm{H}}$ signals from topotecan $3^{+}$(red in Fig. 4A) experienced a greater magnetic shielding ( $\Delta \delta \sim 0.3-0.4 \mathrm{ppm}$, red in Fig. 4A) than its other nuclei to denote the lactone but also dimethyl ammonium portions of the drug residing in the host's aromatic cavity. The method of continuous variation (Fig. 4B) indicated the formation of a binary $\mathbf{1}^{6-} \subset \mathbf{3}^{+}$complex, while the fluorescence binding isotherm (Fig. 4C; see also Fig. S10 $\dagger$ ) fits well to the $1: 1$ stoichiometric model with a random distribution of 
(A)

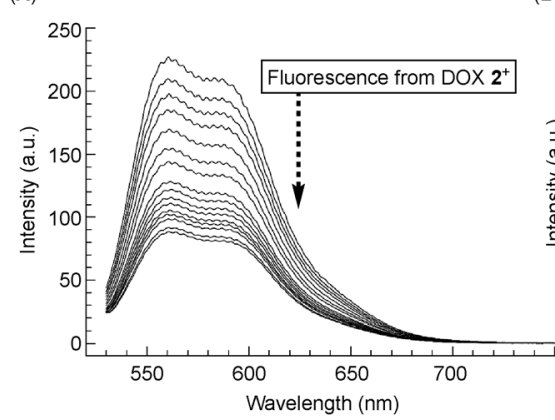

(D)

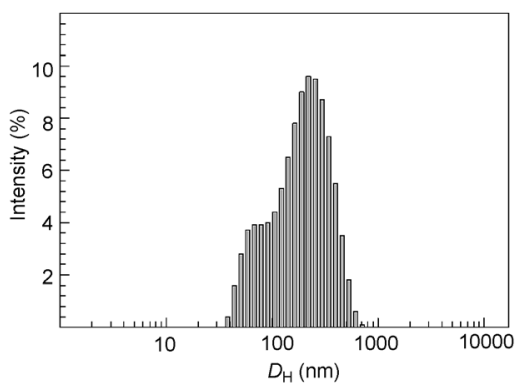

(B)

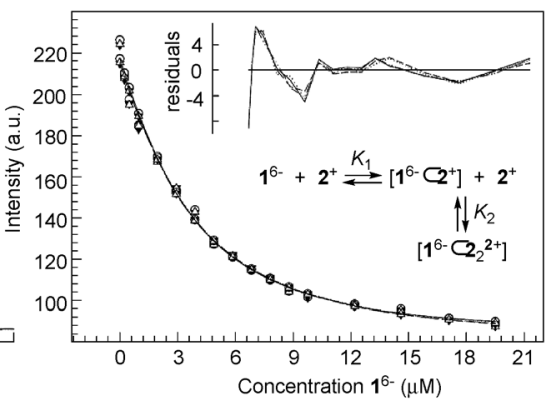

(E)

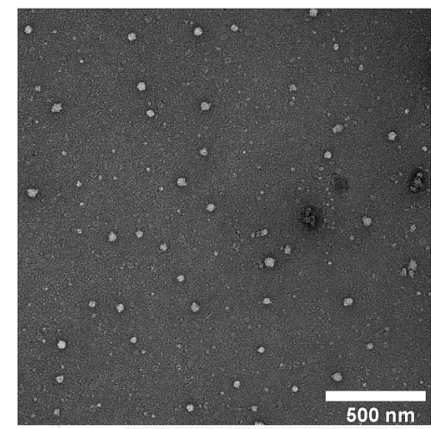

(C)

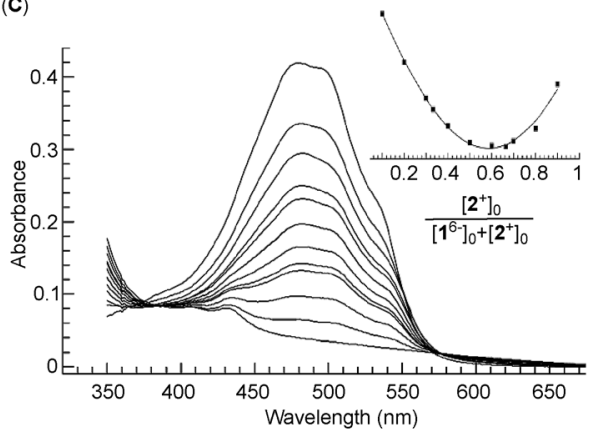

(F)

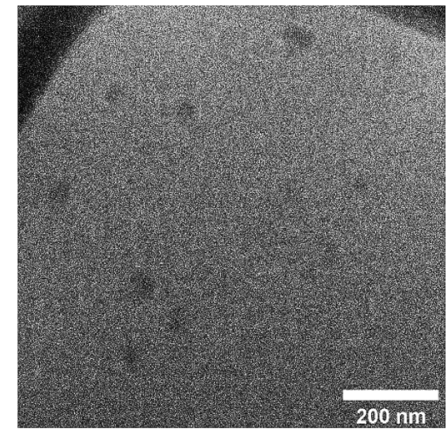

Fig. 3 (A) An incremental addition of basket $1^{6-}$ to a $1.0 \mu \mathrm{M}$ solution of doxorubicin $2^{+}(10 \mathrm{mM}$ PBS at $\mathrm{pH}=7.0)$ was monitored with fluorescence spectroscopy $\left(\lambda_{\mathrm{ex}}=500 \mathrm{~nm} ; 298 \mathrm{~K}\right)$. (B) A change in the emission intensity of $2^{+}$as a function of the increasing concentrations of $1^{6-}$ was subjected to global $\left(550-590 \mathrm{~nm}\right.$ ) nonlinear regression analysis using a $2: 1$ stoichiometric model (note that doxorubicin $2^{+}$is in this experimental setup virtually acting as a host with the stoichiometry depicted as $2: 1$ ); the reported $K_{1}=3.2 \pm 0.8 \times 10^{5} \mathrm{M}^{-1}$ and $K_{2}=9 \pm 1 \times 10^{6} \mathrm{M}^{-1}$ represent the arithmetic mean and standard deviation from two independent measurements (Fig. S $8 \dagger$ ); a random distribution of residuals is shown in the inset. (C) UV-Vis spectra of $0.04 \mathrm{mM}$ doxorubicin $2^{+}(10 \mathrm{mM}$ PBS at pH $=7.0)$ in the presence of different proportions of $1^{6-}$ with the Job plot (inset) obtained using the absorbance at $500 \mathrm{~nm}$; for the $y$ axis: $\left(A_{\mathrm{obs}}-A_{0}\right)\left[2^{+}\right]_{0}$. (D) The size distribution of particles in the solution of $1^{6-}$ $(0.05 \mathrm{mM})$ and $2^{+}(0.1 \mathrm{mM})(10 \mathrm{mM}$ PBS at $\mathrm{pH}=7.0)$ was obtained from DLS measurement at $298.0 \mathrm{~K}$. (E) TEM image of a $10 \mathrm{mM}$ PBS solution of $1^{6-}(0.1 \mathrm{mM})$ and $2^{+}(0.2 \mathrm{mM})$ at $\mathrm{pH}=7.0$ deposited on a copper grid and stained with uranyl acetate. (F) Cryo-TEM image of a $0.1 \mathrm{mM}$ solution of basket $1^{6-}$ containing $2^{+}(0.2 \mathrm{mM})$ in $10 \mathrm{mM}$ PBS at $\mathrm{pH}=7.0$

residuals $\left(K_{1}=2.12 \pm 0.01 \times 10^{4} \mathrm{M}^{-1}\right) ;^{38}$ note that the fluorescence of topotecan $3^{+}$was, as in the case of doxorubicin, quenched by the basket. At last, the results from DLS

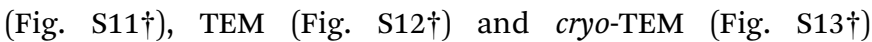
measurements were consistent with $1^{6-} \subset 3^{+}$assembling into circa $35 \mathrm{~nm}$ nanoparticles with the morphology akin to $\mathbf{1}^{6-}$ and $\mathbf{1}^{6-} \subset \mathbf{2}_{2}{ }^{2+}$ nanoparticles.

Why is the encapsulation of doxorubicin characterized with positive cooperativity $(\alpha=112)$ while topotecan prefers to occupy a single pocket of dual-cavity $\mathbf{1}^{6-}$ (i.e. negative cooperativity $\alpha \ll 1) ?^{21,24}$ In order to elucidate the origin of the allostery, we first subjected $1^{6-} \subset 2^{+}$and $1^{6-} \subset 2_{2}{ }^{2+}$ complexes to the Monte-Carlo conformational search using the OPLS3e force field $^{\mathbf{4 0}}$ in implicit water solvent. The global minimum was, in all cases, assessed by running multiple calculations, each starting with the drug assuming a different pose in the basket's cavity (see the ESI $\dagger$ ). The conformational sampling of $1^{6-} \subset 2^{+}$ returned numerous yet similar conformers populating the equilibrium (<1.5 kcal mol ${ }^{-1}$, Fig. $\left.5 \mathrm{~A}\right)$ : all structures encompass drug $2^{+}$docked within the host's top cavity and the methoxybenzene ring poised to form $\mathrm{C}-\mathrm{H} \cdots \pi$ (D, Fig. 5A) and $\pi-\pi$ stacking (E, Fig. 5A) interactions with the surrounding aromatic box. Moreover, three GABA carboxylates from basket $\mathbf{1}^{6-}$ form two hydrogen bonds $\mathbf{A} / \mathbf{B}$ and salt bridge $\mathbf{C}$ with the drug. While the occupied cavity snugs the guest by reducing the average imide $d_{\mathrm{N}-\mathrm{N}}$ distance to 9.19-9.30 $\AA$ (arithmetic mean of three $d_{\mathrm{N}-\mathrm{N}}$ distances in Fig. 5A), the unoccupied (bottom) cavity responds by flexing ${ }^{25 a, 41}$ its phthalimide arms to $d_{\mathrm{N}-\mathrm{N}}=9.92-$ $10.01 \AA$ (Fig. 5D; for empty $1^{6-}, d_{\mathrm{N}-\mathrm{N}}=9.71-9.77 \AA$ ). For the doubly populated $\mathbf{1}^{6-} \subset \mathbf{2}_{2}{ }^{2+}$, the drug molecule docked in the top cavity preserves all five noncovalent contacts $\mathbf{A}-\mathbf{E}$ with the cavity $1^{6-} \subset 2_{2}{ }^{2+}$ (Fig. 5B). Doxorubicin residing in the bottom cavity, though, assumes an equivalent position to that in the top one with three GABA residues forming hydrogen bonds $\mathbf{A}$ and $\mathbf{B}$ and the salt bridge $\mathbf{C}$ to hold it in its place. The transition of singly $\mathbf{1}^{6-} \subset 2^{+}$into doubly populated $1^{6-} \subset 2_{2}{ }^{2+}$ (Fig. 5C) is accompanied by the top cavity retaining its size $d_{\mathrm{N}-\mathrm{N}}=9.20-$ 9.30 ̊ and the bottom one shrinking to $d_{\mathrm{N}-\mathrm{N}}=9.07-9.27 \AA$. The second binding event thus necessitates a reduction in the size of the bottom cavity while concurrently maintaining the shape of the top one (Fig. 5D). We reason that such increase in the steric strain $\left(\Delta H^{\circ}>0\right)$ following the second complexation is likely overcompensated by favourable noncovalent $\mathbf{A}-\mathbf{E}$ contacts $\left(\Delta H^{\circ}\right.$ $\ll 0)$.

The formation of binary $1^{6-} \subset 2^{+}$is a favourable process $\left(K_{1}=\right.$ $3.2 \pm 0.8 \times 10^{5} \mathrm{M}^{-1}$ ) supported with the notion that the basket's 
(A)

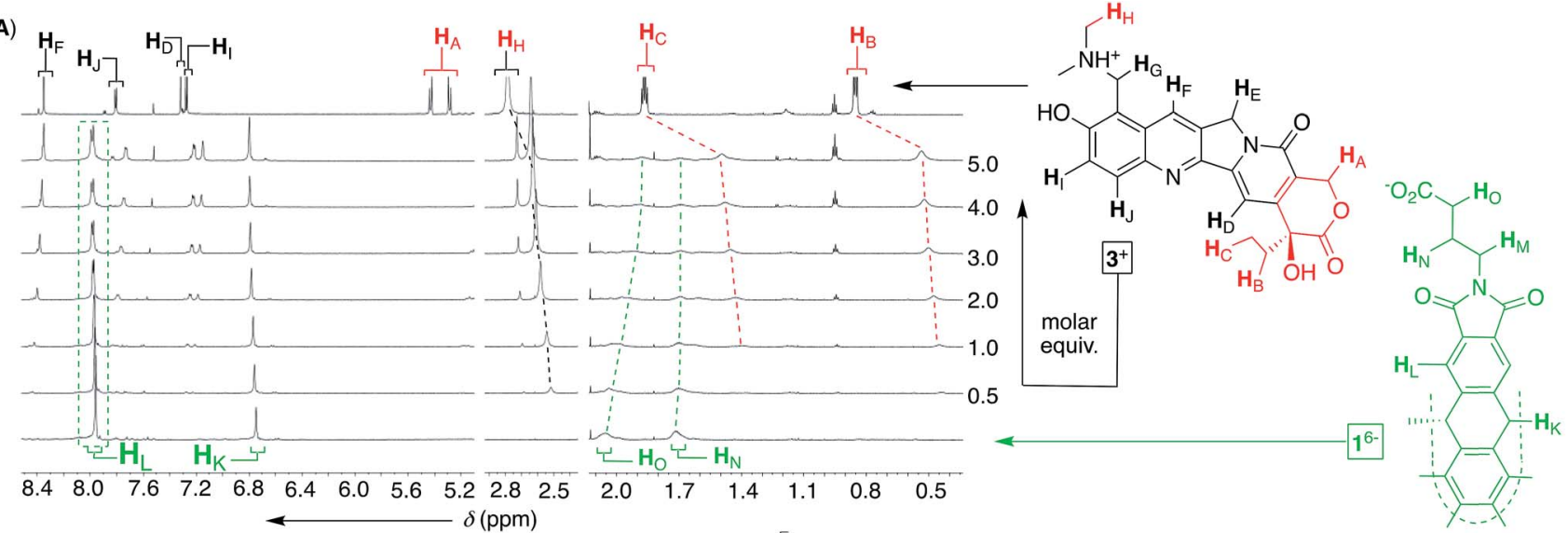

(B)

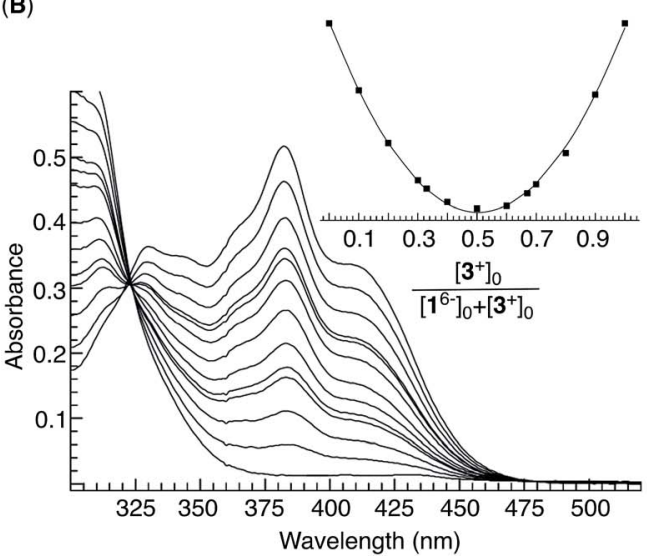

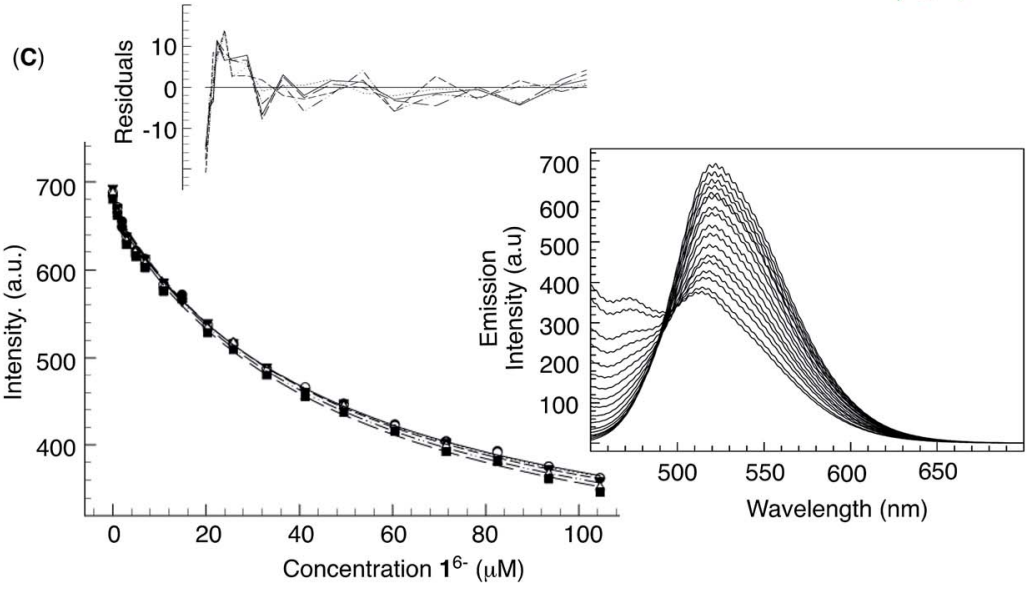

Fig. 4 (A) ${ }^{1} \mathrm{H}$ NMR spectra $\left(850 \mathrm{MHz}, 298 \mathrm{~K}\right.$ ) depicting an incremental addition of topotecan $3^{+}$(red, top) to $0.1 \mathrm{mM}$ basket $1^{6-}$ (green, bottom) in $10 \mathrm{mM}$ PBS at $\mathrm{pH}=7.0$. (B) UV-Vis spectra of $0.03 \mathrm{mM}$ topotecan $3^{+}(10 \mathrm{mM} \mathrm{PBS}$ at $\mathrm{pH}=7.0)$ in the presence of different proportions of $1^{6-}$ with the Job plot (inset) obtained using the absorbance at $382 \mathrm{~nm}$; for the $y$ axis: $\left(A_{\text {obs }}-A_{0}\right)\left[3^{+}\right]_{0}$. (C) A change in the emission intensity of $3^{+}(2 \mu M$ in $10 \mathrm{mM}$ PBS at $\mathrm{pH}=7.0$ ) as a function of the increasing concentrations of $1^{6-}$ was subjected to global (520-540 nm) nonlinear regression analysis using a $1: 1$ stoichiometric model to give $K_{1}=2.12 \pm 0.01 \times 10^{4} \mathrm{M}^{-1}$, representing the arithmetic mean and standard deviation from two independent measurements (Fig. S10†); a random distribution of residuals is shown in the inset. (Right) Fluorescence spectra of $2 \mu M 3^{+}$in the presence of increasing concentrations of $1^{6-}\left(\lambda_{\mathrm{ex}}=420 \mathrm{~nm} ; 298 \mathrm{~K}\right)$ in $10 \mathrm{mM} \mathrm{PBS}$ at $\mathrm{pH}=7.0$.

top grips onto the drug by using all three of its phthalimide arms and engaging in five favourable non-covalent interactions (Fig. 5A): the top cavity shrinks to hold the drug, while the bottom one expands; the hydrophobic effect is expected to contribute to this complexation as well. ${ }^{42}$ With all three of the top cavity's sides being "tied", the bottom three phthalimides become more rigid ${ }^{25 a}$ and thus more preorganized to, via favourable entropy, increase the affinity for the second drug molecule $\left(K_{2}=9 \pm 1 \times 10^{6} \mathrm{M}^{-1}\right)$. That is to say, the first complexation preorganizes the host for the second one to occur with a minimal loss in entropy, and gain in enthalpy and the overall positive homotopic cooperativity. The role of the assembly of $\mathbf{1}^{6-} \subset \mathbf{2}_{2}{ }^{2+}$ has not been taken into consideration although it is more than likely that the process has an effect on the cooperativity and the mechanism by which the recognition takes place.

A validation for our computational findings comes from the results of ${ }^{1} \mathrm{H}$ NMR spectroscopic experiments (Fig. 2A). The methoxybenzene nuclei from doxorubicin $2^{+}$sustained the greatest degree of magnetic perturbation to implicate it occupying the host's cavities as the theory suggests (Fig. 5).

The Monte-Carlo conformational sampling of $1^{6-} \subset 3^{+}$ (Fig. 6) revealed topotecan docking in the basket's cavity by anchoring its lactone ring (Fig. 6A) or dimethyl ammonium group in the aromatic box (Fig. 6B). The apparent absence of additional noncovalent contacts between $3^{+}$and $\mathbf{1}^{6-}$ (except one hydrogen bond $\mathbf{A}$ in Fig. $6 \mathrm{~B}$ ) makes this host-guest pair less complementary than the prior one. The basket is thus loosely held by topotecan to make the $1^{6-} \subset 3^{+}$complex less preorganized for binding another molecule of the drug. Moreover, a weaker complexation could also be alleged to provide insufficient driving force for overcoming the strain required in the second binding event. In support of topotecan $3^{+}$being less complementary to dual-cavity $\mathbf{1}^{6-}$ than doxorubicin $2^{+}$, we note that binary $\mathbf{1}^{6-} \subset 3^{+}$is an order of magnitude less stable $\left(K_{1}=\right.$ $\left.2.12 \pm 0.01 \times 10^{4} \mathrm{M}^{-1}\right)$ than $\mathbf{1}^{6-} \subset 2^{+}\left(K_{1}=3.2 \pm 0.8 \times 10^{5} \mathrm{M}^{-1}\right)$. On another note, the results of the NMR titration (Fig. 4A) showed that protons at both termini of topotecan became 
(A)

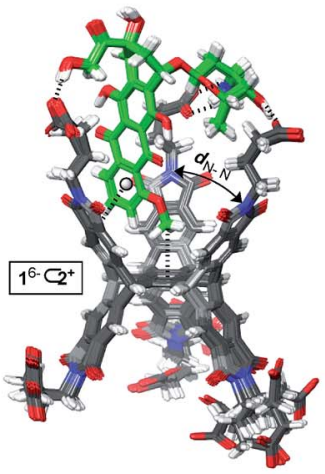

(B)

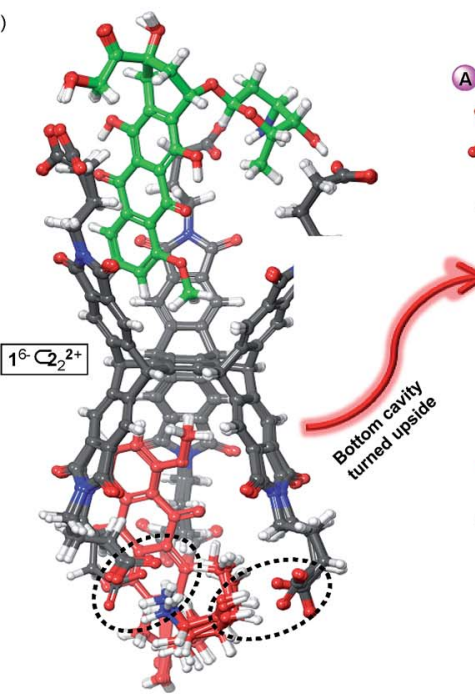

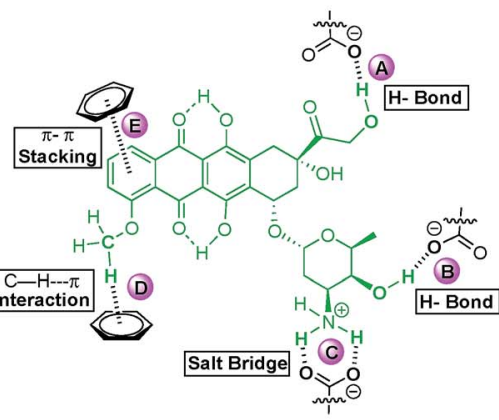

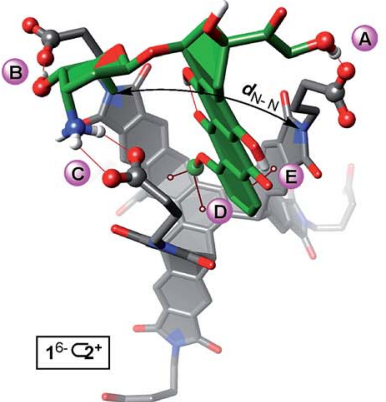

(C)

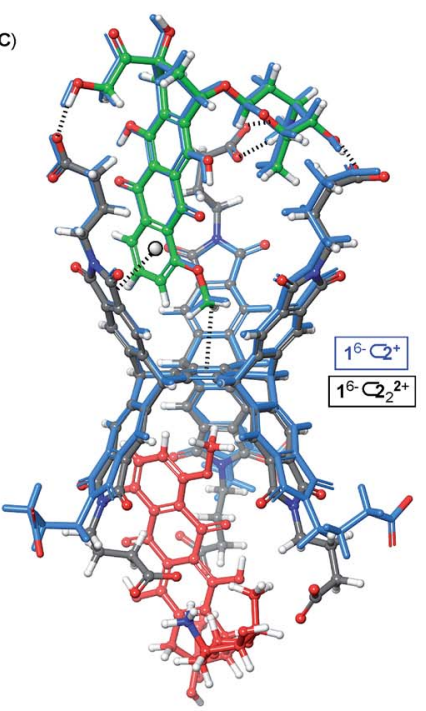

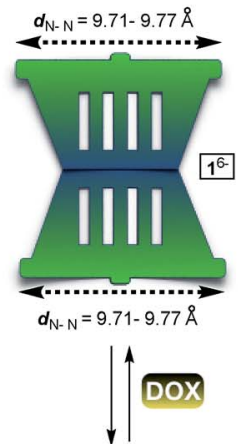

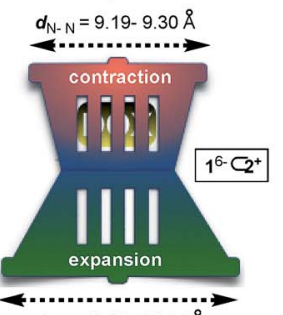

$d_{N-N}=9.92-10.01 \AA$

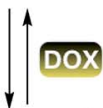

$\boldsymbol{d}_{\mathrm{N}-\mathrm{N}}=9.20-9.30 \AA$

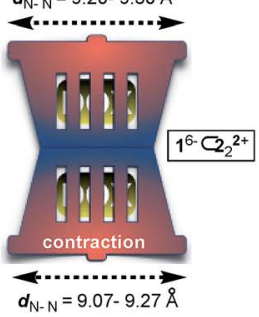

Fig. 5 (A) A side view of 155 conformers of $1^{6-} \subset 2^{+}\left(<1.5 \mathrm{kcal} \mathrm{mol}^{-1}\right)$ obtained by the Monte-Carlo conformational search (OPLS3e, Schrodinger) in implicit water. (Middle) Chemical structure of doxorubicin $2^{+}$forming favourable A-E noncovalent contacts with basket $1^{6-}$. (Right) Top view of the most stable conformer of $1^{6-} \subset 2^{+}$. (B) A side view of 33 conformers of $1^{6-} \subset 22^{2+}\left(<1.5 \mathrm{kcal} \mathrm{mol}^{-1}\right)$ obtained by the Monte-Carlo conformational search (OPLS-3, Schrodinger) in implicit water. (Right) The most stable conformer of $1^{6-} \subset 2^{+}$showing DOX $2^{+}$(red) forming favourable A-E contacts with the basket. (C) The most stable conformers of $1^{6-} \subset 2^{+}$(blue) and $1^{6-} \subset 2_{2}{ }^{2+}$ are aligned to demonstrate the induced-fit mode of binding in the bottom cavity. (D) A schematic representation of the induced-fit model of binding of doxorubicin $2^{+}$with dual-cavity $1^{6-}$
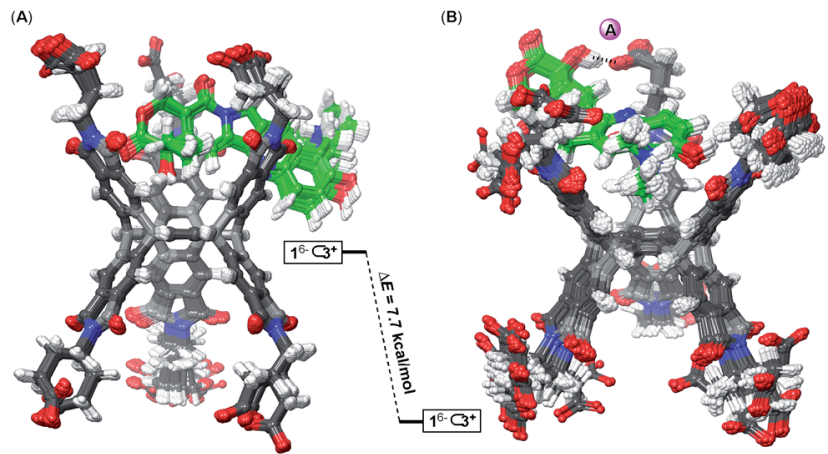

Fig. 6 Side views of 467 (A) and 271 (B) conformers of $1^{6-} \subset 3^{+}(<1.5$ $\mathrm{kcal} \mathrm{mol}^{-1}$ ) obtained by the Monte-Carlo conformational search (OPLS3e, Maestro) in implicit water. Note that topotecan $3^{+}$inserts (A) its lactone ring or (B) dimethyl ammonium group in the cavity of basket $1^{6-} . \Delta E$ represents the computed difference in steric energies of two most stable conformers in $(A)$ and $(B)$. magnetically shielded to, perhaps, indicate the concurrent existence of both computed poses shown in Fig. 6 .

\section{Conclusions}

We discovered an abiotic host capable of encapsulating two molecules of anticancer drug doxorubicin in a positive cooperative fashion with ternary drug-host complexes assembling into nanoparticles. The origin of the cooperativity can, in part, be traced back to extraordinary complementarity of the drug to the abiotic receptor in addition to its preorganization. That is to say, at least ten favorable noncovalent contacts are established between doxorubicin and the dual-cavity basket, resembling those found for receptors in the natural world. The basket acts as a "claw grabber" capturing drug molecules with its six "sticky carboxylate fingers" and snugging them within the adjoining aromatic cavities in an induced-fit manner. In the case of topotecan, however, we posited that the paucity of favorable 
noncovalent contacts between the basket and the drug, i.e. lack of complementarity, played the key role in the observed complexation stoichiometry. In general, our discovery sets the stage for (a) improving our fundamental understanding of cooperativity in artificial systems and (b) creating a family of novel hosts capable of complexing plane-shaped anticancer agents with high affinity and selectivity.

Holding a high drug payload $(41 \%),{ }^{13}$ nanoparticles release doxorubicin at a lower $\mathrm{pH}^{\mathbf{4 3}}$ (Fig. S14/S15 $\dagger$ ) or in the presence of spermine $^{44}$ (Fig. S16 $\dagger$ ), as signaled by the increase in fluorescence. ${ }^{36 b, 45}$ Accordingly, our aims shift to the examination of this soft material for delivery ${ }^{\mathbf{4 6}}$ as well as scavenging ${ }^{47}$ of anticancer drugs in biological and other competitive media. ${ }^{22}$ In fact, with dual-cavity baskets amenable to screening protocols, ${ }^{30}$ one should be able to optimize their capacity for selective removal $^{48}$ as well as amplified delivery ${ }^{22}$ of targeted pharmaceuticals.

\section{Conflicts of interest}

There are no conflicts to declare.

\section{Acknowledgements}

This work was financially supported with funds obtained from the Army Research Office (W911NF-17-1-0140). We would like to thank Prof. C. M. Hadad from the Ohio State University, Department of Chemistry and Biochemistry, for useful suggestions and inspiring discussions.

\section{Notes and references}

1 (a) W. C. W. Chan, Acc. Chem. Res., 2017, 50, 627; (b) F. Caruso, T. Hyeon and V. M. Rotello, Chem. Soc. Rev., 2012, 41, 2537.

2 (a) S. Aftab, A. Shah, A. Nadhman, S. Kurbanoglu, S. Aysil Ozkan, D. D. Dionysiou, S. S. Shukla and T. M. Aminabhavi, Int. J. Pharm., 2018, 540, 132; (b) R. Awasthi, A. Roseblade, P. M. Hansbro, M. J. Rathbone, K. Dua and M. Bebawy, Curr. Drug Targets, 2018, 19, 1696; (c) T. J. Anchordoquy, Y. Barenholz, D. Boraschi, M. Chorny, P. Decuzzi, M. A. Dobrovolskaia, Z. S. Farhangrazi, D. Farrell, A. Gabizon, H. Ghandehari, B. Godin, N. M. La-Beck, J. Ljubimova, S. M. Moghimi, L. Pagliaro, J.-H. Park, D. Peer, E. Ruoslahti, N. J. Serkova and D. Simberg, ACS Nano, 2017, 11, 12; (d) L. Isaacs, Adv. Drug Delivery Rev., 2012, 64, 763; (e) C. M. Hartshorn, M. S. Bradbury, G. M. Lanza, A. E. Nel, J. Rao, A. Z. Wang, U. B. Wiesner, L. Yang and P. Grodzinski, ACS Nano, 2018, $12,24$.

3 S. Wilhelm, A. J. Tavares, Q. Dai, S. Ohta, J. Audet, H. F. Dvorak and W. C. W. Chan, Nat. Rev. Mater., 2016, 1, 16014.

4 C. Kinnear, T. L. Moore, L. Rodriguez-Lorenzo, B. RothenRutishauser and A. Petri Fink, Chem. Rev., 2017, 117, 11476. 5 V. P. Torchilin, Handb. Exp. Pharmacol., 2010, 197, 3.
6 R. Mout, F. Moyano Daniel, S. Rana and M. Rotello Vincent, Chem. Soc. Rev., 2012, 41, 2539.

7 Y.-R. Zheng, K. Suntharalingam, T. C. Johnstone and S. J. Lippard, Chem. Sci., 2015, 6, 1189.

8 K. Uekama, F. Hirayama and T. Irie, Chem. Rev., 1998, 98, 2045.

9 M. Giuliani, I. Morbioli, F. Sansone and A. Casnati, Chem. Commun., 2015, 51, 14140.

10 L. Cao, G. Hettiarachchi, V. Briken and L. Isaacs, Angew. Chem., Int. Ed., 2013, 52, 12033.

11 X. Ma and Y. Zhao, Chem. Rev., 2015, 115, 7794.

12 L. Wang, L.-l. Li, Y.-s. Fan and H. Wang, Adv. Mater., 2013, 25, 3888.

13 K. Park, ACS Nano, 2013, 7, 7442.

14 C. Weeden, K. J. Hartlieb and L. Y. Lim, J. Pharm. Pharmacol., 2012, 64, 1403.

15 D. Ma, G. Hettiarachchi, D. Nguyen, B. Zhang, J. B. Wittenberg, P. Y. Zavalij, V. Briken and L. Isaacs, Nat. Chem., 2012, 4, 503.

16 W. Shao, X. Liu, G. Sun, X.-Y. Hu, J.-J. Zhu and L. Wang, Chem. Commun., 2018, 54, 9462.

17 A. Abdul Karim, Q. Dou, Z. Li and X. J. Loh, Chem.-Asian J., 2016, 11, 1300.

18 C. A. Hunter and H. L. Anderson, Angew. Chem., Int. Ed., 2009, 48, 7488.

19 Y. Li, T. Zhao, C. Wang, Z. Lin, G. Huang, J. Gao, C. Wang and D. Sumer Baran, Nat. Commun., 2016, 7, 13214.

20 J. A. Riddle, X. Jiang and D. Lee, Analyst, 2008, 133, 417.

21 S. Shinkai, M. Ikeda, A. Sugasaki and M. Takeuchi, Acc. Chem. Res., 2001, 34, 494.

22 Y. Li, Y. Wang, G. Huang and J. Gao, Chem. Rev., 2018, 118, 5359.

23 K. C. Tjandra and P. Thordarson, Bioconjugate Chem., 2019, 30, 503.

24 J. D. Badjic, A. Nelson, S. J. Cantrill, W. B. Turnbull and J. F. Stoddart, Acc. Chem. Res., 2005, 38, 723.

25 (a) S. Chen, M. Yamasaki, S. Polen, J. Gallucci, C. M. Hadad and J. D. Badjic, J. Am. Chem. Soc., 2015, 137, 12276; (b) S. Chen, L. Wang, S. M. Polen and J. D. Badjic, Chem. Mater., 2016, 28, 8128.

26 J. B. Wittenberg and L. Isaacs, Supramol. Chem.: Mol. Nanomater., 2012, 1, 25.

27 K. Hermann, M. Nakhla, J. Gallucci, E. Dalkilic, A. Dastan and J. D. Badjic, Angew. Chem., Int. Ed., 2013, 52, 11313.

28 P. Thordarson, Chem. Soc. Rev., 2011, 40, 1305.

29 S. Mitragotri and P. Stayton, MRS Bull., 2014, 39, 219.

30 T. Neal, W. Wang, L. Zhiquan, R. Peng, P. Soni, H. Xie and J. D. Badjic, Chem.-Eur. J., 2019, 25, 1115.

31 L. Zhiquan, S. Polen, C. M. Hadad, T. V. RajanBabu and J. D. Badjic, J. Am. Chem. Soc., 2016, 138, 8253.

32 Y. Ruan, H. A. Taha, R. J. Yoder, V. Maslak, C. M. Hadad and J. D. Badjic, J. Phys. Chem. B, 2013, 117, 3240.

33 L. Zhiquan, H. Xie, S. E. Border, J. Gallucci, R. Z. Pavlovic and J. D. Badjic, J. Am. Chem. Soc., 2018, 140, 11091.

34 (a) S. E. Border, R. Z. Pavlovic, L. Zhiquan, M. J. Gunther, H. Wang, H. Cui and J. D. Badjic, Chem. Commun., 2019, 
DOI: 10.1039/c8cc08938h; (b) L. Wang, T. Neal, S. Chen and J. D. Badjic, Chem.-Eur. J., 2017, 23, 8829.

35 S. Chen, Y. Ruan, J. D. Brown, J. Gallucci, V. Maslak, C. M. Hadad and J. D. Badjic, J. Am. Chem. Soc., 2013, 135, 14964.

36 (a) S. Chen, S. M. Polen, L. Wang, M. Yamasaki, C. M. Hadad and J. D. Badjic, J. Am. Chem. Soc., 2016, 138, 11312; (b) W.-C. Geng, S. Jia, Z. Zheng, Z. Li, D. Ding and D.-S. Guo, Angew. Chem., Int. Ed., 2019, DOI: 10.1002/anie.201813397.

37 D.-S. Guo, V. D. Uzunova, X. Su, Y. Liu and W. M. Nau, Chem. Sci., 2011, 2, 1722.

38 F. Ulatowski, K. Dabrowa, T. Balakier and J. Jurczak, J. Org. Chem., 2016, 81, 1746.

39 I. Saha, J. H. Lee, H. Hwang, T. S. Kim and C.-H. Lee, Chem. Commun., 2015, 51, 5679.

40 E. Harder, W. Damm, J. Maple, C. Wu, M. Reboul, J. Y. Xiang, L. Wang, D. Lupyan, M. K. Dahlgren, J. L. Knight, J. W. Kaus, D. S. Cerutti, G. Krilov, W. L. Jorgensen, R. Abel and R. A. Friesner, J. Chem. Theory Comput., 2016, 12, 281.
41 P. Thordarson, E. J. A. Bijsterveld, J. A. A. W. Elemans, P. Kasak, R. J. M. Nolte and A. E. Rowan, J. Am. Chem. Soc., 2003, 125, 1186.

42 L. Zhiquan, S. M. Polen, C. M. Hadad, T. V. RajanBabu and J. D. Badjic, Org. Lett., 2017, 19, 4932.

43 (a) J.-Z. Du, H.-J. Li and J. Wang, Acc. Chem. Res., 2018, 51, 2848; (b) B. Li, Z. Meng, Q. Li, X. Huang, Z. Kang, H. Dong, J. Chen, J. Sun, Y. Dong, J. Li, X. Jia, J. L. Sessler, Q. Meng and C. Li, Chem. Sci., 2017, 8, 4458.

44 Y. Chen, Z. Huang, H. Zhao, J.-F. Xu, Z. Sun and X. Zhang, ACS Appl. Mater. Interfaces, 2017, 9, 8602.

45 Y. Yuan and B. Liu, Chem. Sci., 2017, 8, 2537.

46 D. Bousmail, L. Amrein, J. J. Fakhoury, H. H. Fakih, J. C. C. Hsu, L. Panasci and H. F. Sleiman, Chem. Sci., 2017, 8, 6218.

47 H. J. Oh, M. S. Aboian, M. Y. J. Yi, J. A. Maslyn, W. S. Loo, X. Jiang, D. Y. Parkinson, M. W. Wilson, T. Moore, C. R. Yee, G. R. Robbins, F. M. Barth, J. M. DeSimone, S. W. Hetts and N. P. Balsara, ACS Cent. Sci., 2019, DOI: 10.1021/acscentsci.8b00700.

48 J.-C. Leroux, Nat. Nanotechnol., 2007, 2, 679. 